\title{
6G Networks Orientation by Quantum Mechanics
}

\author{
Paulo Sergio Rufino Henrique* and Ramjee Prasad \\ CTIF Global Capsule (CGC), Department of Business Development and \\ Technologies, Aarhus University, Herning, Denmark \\ E-mail:rufino@spideo.tv; ramjee@btech.au.dk \\ ${ }^{*}$ Corresponding Author
}

Received 29 July 2021; Accepted 05 December 2021;

Publication 11 February 2022

\begin{abstract}
Quantum mechanics is a part of physics that studies the interactions of matter, light, and particles at the atomic and subatomic levels. Since its initial concepts in the early 1900s built upon extensive research of Nobel laureates such as Max Planck, Niels Bohr, Albert Einstein, and Richard Feynman, amongst others to the first proposed quantum computers by Paul Benioff in 1980, the concept of quantum technologies has evolved. Two central studies derived from quantum mechanics that can support and revolutionize future wireless technologies are quantum computers and quantum communications. The investigation for building the next generation of wireless networks has begun. Therefore, many technological opportunities for applying innovative solutions and advanced concepts are on the table as an option to unlock the full potential of $6 \mathrm{G}$ for providing an intelligent, superfast, and secure network. Having said that, quantum mechanics come into play to offer a breakthrough opportunity that will change the world since the popularization of the Internet, and it will propel $6 \mathrm{G}$ use cases to be remarkably successful, but only if quantum physics can be engineered and converged into the forementioned quantum technologies to support the achievement of Society 5.0.
\end{abstract}

Journal of ICT Standardization, Vol. 10_1, 39-62.

doi: 10.13052/jicts2245-800X.1013

(C) 2022 River Publishers 
Therefore, overcoming the quantum challenges, $6 \mathrm{G}$ can benefit in many ways. One of them is Quantum computing (QC) that will surpass the computational capabilities of classic computers limited by binary transactions known as bits to resolve future challenges using quantum states to process information in quantum bits (Qubits). Correspondingly, quantum computing will merge with Artificial Intelligence (AI) to create a new model known as Quantum Machine Learning (QML) to deal with the exponential growth of Big Data faster than any existing computational model. In Addition, quantum communications will deliver a safer network, utilizing Quantum Key Distribution (QKD) and inaugurate the next generation of the Internet, much safer for all. Thus, this paper presents a holistic overview of Quantum as a service (QaaS) as a future deployment in the $6 \mathrm{G}$ architecture, but only if quantum technologies can be mastered in the next upcoming years. Most likely that QaaS will become available for commercial purposes by the hyperscalers, the ones able to cope with the total cost of ownership (TCO) of these state of art technologies.

Keywords: 6G, AI, electromagnetic radiation, quantum computing, quantum mechanics, quantum communications infrastructure (QCI), quantum machine learning, quantum key distribution (QKD), QaaS, TCO, big data.

This paper is divided by an introduction that summarizes the foundation of quantum mechanics, its laws, and the researchers' pioneers responsible for setting the principles used to create the next generation of quantum technologies. In Section 2, there is a presentation of the core parts of quantum physics used to create quantum computers and quantum communications services, which includes the technological challenges to mature these technologies. Finally, in Section 3, the conclusions are presented.

\section{Introduction to Quantum Mechanics}

Quantum computing is not a new science [1], and its objectives are to deploy a new generation of computers that can exponentially exceed the classical computing power and unlock a new myriad of services and innovative solutions to humanity. On quantum technologies and primarily in quantum computing, there were several pioneers to be named as such innovators for contributing to utilize quantum mechanics to create a new computational architecture to overcome the current constraints of classical computing based on bits, or simply saying in the combination of only two states of values zero (0) or one (1). Firstly it is essential to revisit the intrinsics dynamics of quantum 
physics before a further explanation is given in how quantum technologies can be applied in 6G Networks. Quantum mechanics is part of the physics science that studies the behavior of light and particles to the atomic and subatomic levels [2]. The theory started gaining force at the beginning of the XX century with the studies about blackbody radiation and how light propagates [3]. This theory did not fit well in classical physics, and it could not be used to describe the behavior of light emission and its absorption in a body. The research carried out during the first decades of the 1900s by renowned scientists Max Karl Ernst Ludwig Planck (1858-1947) and Albert Einstein (1879-1955) [4] demonstrates some strange phenomena occurring with light and matter that escape from the knowledge of classical physics. This mysterious phenomenon was correlated to how a source of energy, considering light, would be emitted and absorbed by a body. At that time, the scientific community could not agree on the definition of how a photoelectric effect would work, mainly if this light emission would be based on particles or waves. Max Planck proposed the quantum theory, which in principle describes that light emission eradiates small packets of energy, known as quanta. Max Planck succeeded in this field thanks to combining previous theorems built previously by other researchers. Planck's predecessors in the quantum field of research were the Nobel laureates Wilhelm Carl Werner Otto Fritz Franz Wien (1864-1928) and John William Strutt (1942-1919), this latter also known as Lord Rayleigh [5].

The quanta theory inaugurated a new chapter that disrupted classical Newtonian physics and gave Planck a Nobel prize in 1918 [6]. Below there is a description of Plancks' equation describing the direct correlation between energy and its radiation.

$$
E=h \cdot V
$$

\section{Planck's equation}

Planck's equation is described by the energy (E) of the radiation source, or electromagnetic wave, known as quanta, which is proportionally related to the frequency of the radiation (v) along with a Planck constant (h). The equations represent the Planck constant as (h), and its value is $\mathbf{6 . 6 2 6} \times \mathbf{1 0}^{\mathbf{3 4}}$ Joule per second. In other words, the Planck theory discovered an association that described the light propagation process and the assimilation of that light into a body. "energy is not emitted or absorbed continuously. It is emitted and absorved in form of wave packets or quanta. In case of light the Quantum 


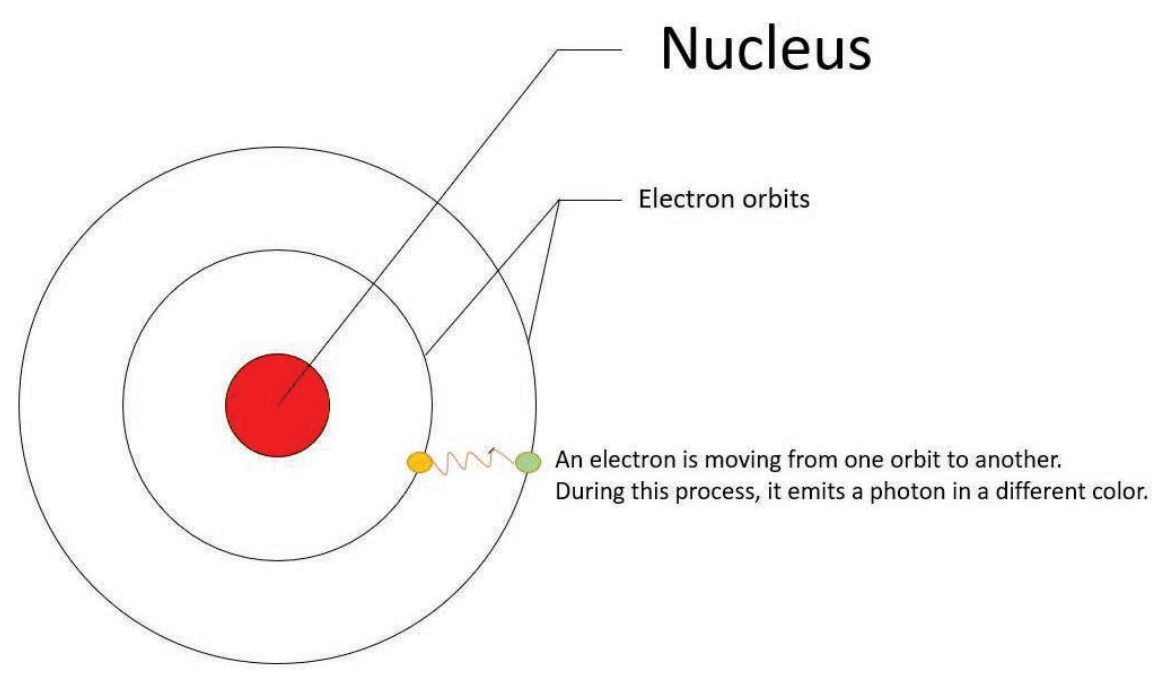

Figure 1 Neils Bohr atomic model - electrons movement and energy emission.

of energy is often called Photon" [7]. However, the Danish physicist Neils Bohr (1885-1962) and Werner Heisenberg (1901-1976) offered continuity to Planck's theory and shocked the world, breaking the concept of the deterministic universe. Neils Bohr won the Nobel Prize in 1922 to explain why atoms irradiate light in specific wavelengths and develop a quantum theory that describes a movement of an atom jumping from an inferior orbit to another higher orbit, which triggers the emission of specific radiation [8]. Figure 1 describes Neils Bohr's atomic model. In this model, every orbit has an integer value, representing a quantum value represented by $(n)$. When an electron goes from an orbit to another, it absorbs or emits a photon in a specific wavelength, characterized by a specific color in the light spectrum, according to the position in the orbit that the electron will take over. Later, Neils also explained that light could behave like waves or particles, depending on the analysis's perspective.

Werner Heisenberg was another physicist to win the Nobel prize in Physics in 1932 for the creation of quantum mechanics [9], in which he emphasized the principle of uncertainty [10], in which an electron could not have its orbit position pre-determined in a specific time period, but only estimated utilizing matrix equations to abstract these values. Therefore, Werner and Neils brought into light quantum physics and its theorems based on probabilities rather than the certainty of a result. The new science caused different reactions, including from the father of the general theory of relativity 
Albert Einstein (1879-1955), who cast doubts about this indeterministic behavior. However, it was later in 1935 that Einstein wrote a paper of four pages long with his colleagues Boris Yakovlevich Podolsky (1896-1966) and Nathan Rosen (1909-1995) entitled "Can Quantum-Mechanical Description of Physical Reality be Considered Complete?" [11] enabled the realization of the quantum computing concepts [12] utilizing the entangled state of particles. In this paper, which is also notoriously known as EPR paper Einstein and his colleagues state an experiment of source that derives two identical particles and their consequences. In summary, once the quantum measurement is made in both particles, both will present the same value. The EPR paper's issue is that this would probably not be possible if the two particles are separated at a more considerable distance, then it would require the particles to send a signal to pair themselves faster than light. In a nutshell, this assumption would break one of the fundamental laws of the theory of Relativity, as nothing can travel faster than light. Einstein defined this observation as a "spooky action", which would require some "hidden variables" to be added in the Quantum Mechanics to describe such event of particles correlations. In summary, the "locality" was impeding the pairing of quantum particles to be measured equally in a far distance and almost instantaneously. This was one of the reasons why the EPR paper challenged the completion of Quantum theory. However, the entanglement of particles was proved later to be correct, and it opened opportunities for the exploration of quantum information and quantum computing utilizing the entanglement state, which cemented one of the last outstanding contributions of Einstein to science and humanity, even though the theory of Relativity is still intact and nothing, for now, can travel faster than light. Figure 2 shows an example of quantum entanglement proposed in the EPR paper.

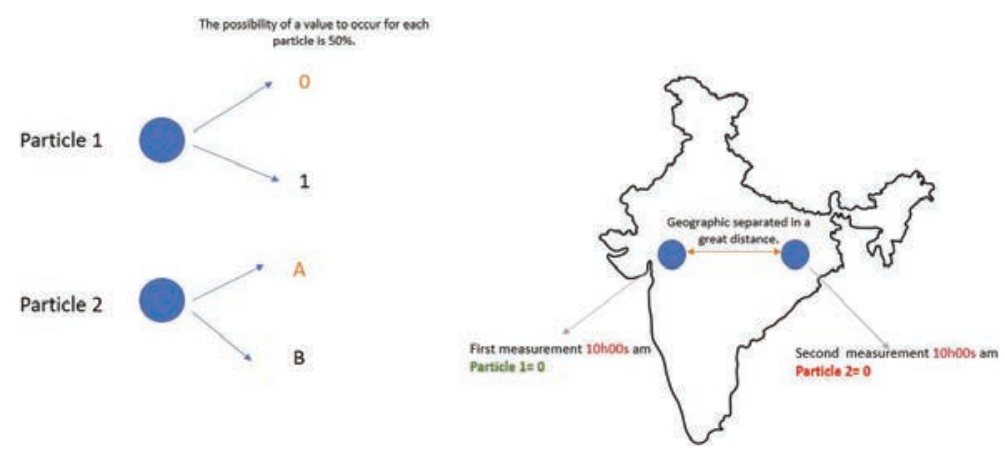

Figure 2 Quantum entanglement of two particles by EPR paper. 
The hidden variables, which could cause the spooky action on the quantum particles correlations, puzzled the scientific community for many years since the EPR paper. However, in 1964 the scientist John Stwart Bell (1928-1990) mathematically proof that there were not possible to have hidden variables to describe the quantum entanglement in the paper entitled "On the Einstein Podolsky Rosen Paradox" [13]. John Bell, who worked for the renowned Conseil Européen pour la Recherche Nucléaire (CERN) and was an admirer of Einstein [14], buried the idea of locality presented in the EPR paper. John Bell proved mathematically that the quantum entanglement was genuine and occurred without an implication of a hidden variable [15]. Therefore, the concept of locality for quantum entanglement dissipated. Nevertheless, the implications of this concept do not erase the fact that nothing can travel faster than light, and secondly, neither proof be feasible to communicate faster than light. Nonetheless, the full description of why and how are the forces behind quantum entanglement are still one of the greatest mysteries of our generation.

\section{Quantum Technologies the Beginning}

The leading researchers that paved the way for the quantum computing concepts to be explored were the Nobel laureates Richard P. Feynman (1918-1988), Julien Schwinger (1918-1994), and Sin-Itiro Tomonaga (1906-1979) [16]. This trio of scientists was responsible for realizing quantum electrodynamics (QED), which opened the doors for Quantum computing systems. The importance of this discovery led to explaining how particles would interact with each other without a need for field forces, as presented in classical physics. In this aspect, QED described the concept of virtual photons expelled from particle $\mathbf{A}$ that interacts with the virtual photons expelled from particle B. Richard Feynman created a simple diagram to explain the high energy collision between particles in the atomic scales, as shown in Figure 3.

Figure 3 above demonstrates the time as a measurement in the vertical axis, and the horizontal axis represents space as a measurement of coordinates. This diagram (e-) vector represents the electrons. The intersecting lines of these electrons represent their momentum in space and time, before and after the interaction of their virtual photons. The virtual photons interactions are represented by the sinusoidal lines in the middle of the diagram. When the virtual photons are fired from an electron, it travels and interacts with the other electron changing the momentum, in which this electron was in the due curse. The importance of QED created the possibility of creating 


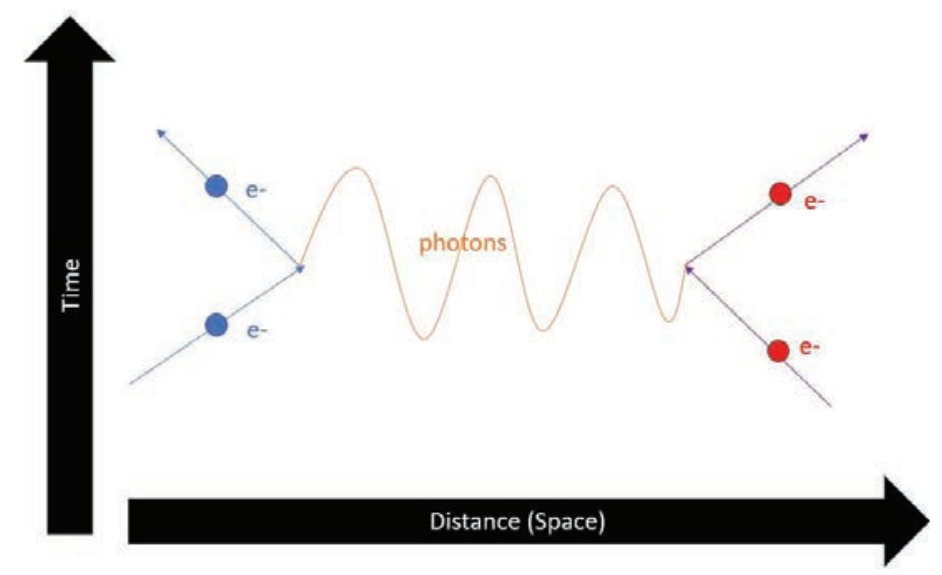

Figure 3 Feynman diagram.

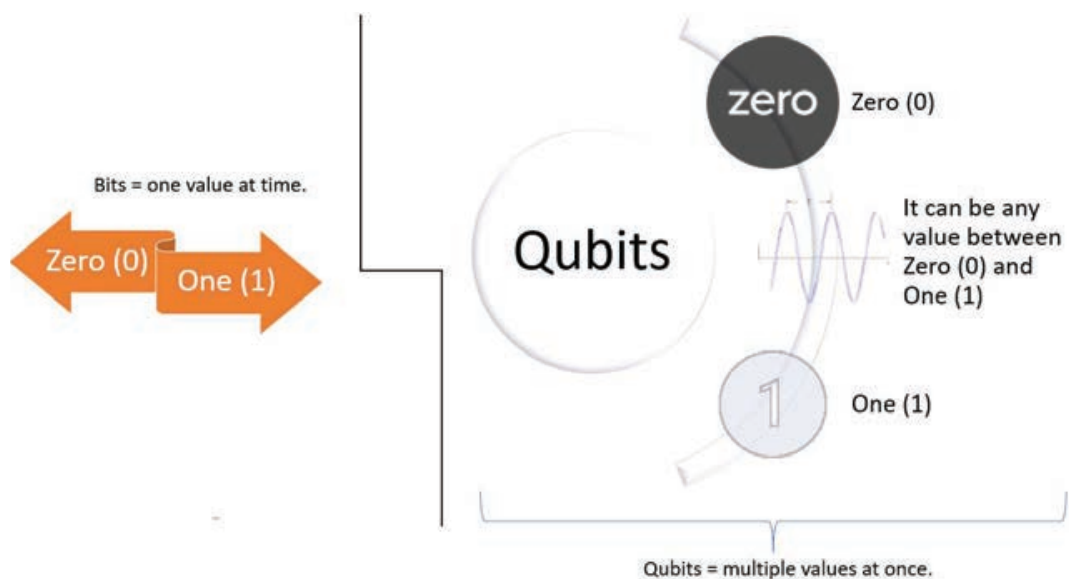

Figure 4 Qubit versus bit.

a Quantum computing architecture that is in its infancy of realization. As one of the fundamental parts of Quantum is the quantum state, as presented earlier. The quantum states in quantum computing represent the probabilities of a particle to present any values while being measured between zero (0) to one (1), which is denominated quantum bits (Qubits). In this situation, the values can be 0,1 , or any value between 0 and 1 simultaneously, as shown in Figure 4.

The Qubits allow more states to propel the computational power against classical computing in an exponential form. Quantum computing, in theory, 
overpass the classical computing power at large; it is based on the possibilities of having more variables as delivered by qubits than a simple binary system in bits. The ability to have simultaneous values in quantum computing is known as being designated in quantum physics as superposition. However, the first to propose a quantum computer was Paul A. Benioff in 1980, using quantum physics to emulate a quantum mechanic computer. This idea was published in his published paper entitled "The Computer as a Physical System: A Microscopic Quantum Mechanical Hamiltonian Model of Computers as Represented by Turing Machines" [17]. Paul Benioff was a pioneer of quantum computing logic and quantum information theory. Paul is a reference for those who want to enter the realms of quantum computation.

For every computing system, there is a need for a computing language. The mathematician Peter Shor [18] was a pioneer in this field when he created the first quantum algorithm known as Shor's algorithm. This new algorithm allowed to factor any prime integer $N$ by its prime number faster than any existing computer using a quantum Fourier as a mathematical methodology. Below there is a technical description of Peter Shor's algorithm factoring processes [19]:

Firstly, Shor's algorithm is distributed in two tasks with clear objectives as defined below:

a. To decrease factoring problems on classical computers

b. To compute the prime numbers using a quantum Fourier transform. Which, this process is computed at a much faster speed applying quantum superposition.

Figure 5 describes the Quantum logical circuit to operate the Shor Algorithm and its sub-routines.

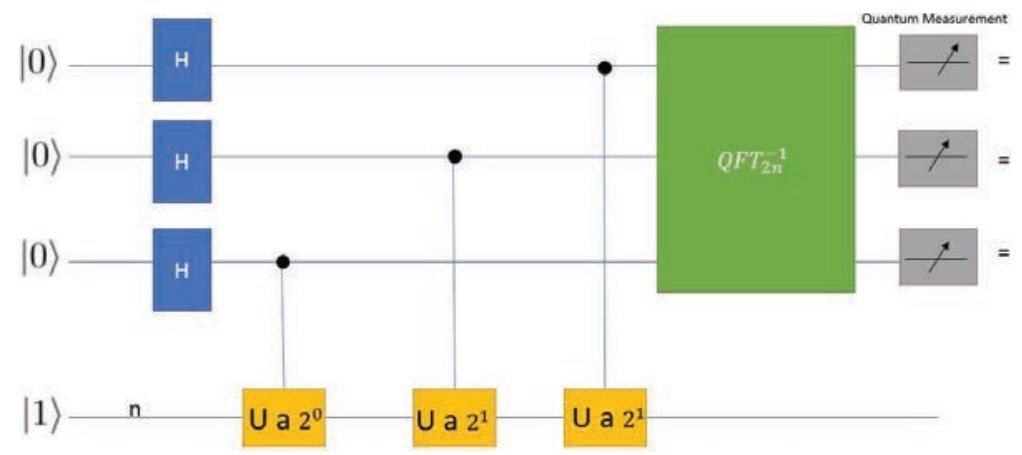

Figure 5 Quantum circuit for Shor's algorithm. 
In the figure shown above, the routine of implementing Shor's algorithm is presented using logical design, which states:

I. Initialize qubits at the zero-position and apply Hadamard Gate.

II. To create the Quantum function (Uf) and apply it to each state after the Hardamad Gate, also known as Quantum Logic Gate, followed by its response.

III. Perform the inverse of the Quantum Fourier Transform.

IV. Obtain the final state of UQFT and reclassify the results.

V. Carry out the quantum measurement

VI. Perform a classical checking to see if the prime number is correctly applying the $a^{S}=1 \bmod N$

\subsection{Quantum Computing Technologies \& Challenges}

Nowadays, the development of quantum computers is still in its infancy. There exist many challenges for developing a standard quantum architecture that can be scaled up commercially for a state-of-the-art quantum computer. Many institutions, organizations, and private sectors invest in quantum computers' research and development ( $\mathrm{R} \& \mathrm{R} \& \mathrm{D})$ of quantum computers. For instance, the European Commission (EC) elaborated the Quantum Technologies Flagship [20], which aims to finance for ten years 1 billion euros to fund quantum computing research for the European Union (EU). Other initiatives in the private sectors to be mentioned are the Quantum AI Campus recently created by Google [21], followed by Microsoft that offers the Azure Quantum Open Cloud [22], and the IBM project IBM Quantum initiative [23]. Due to the earlier described quantum states, there is a possibility to engineer quantum computers to operate quantum superpositions and speed up the computer power, breaking up the linear limits offered by classical computation to offer a new scientific and technological revolution in our planet.

\subsubsection{Challenges for quantum computers technologies}

In terms of challenges, to mention only a few, current quantum computers active requires very low room temperature to operate, which is posed to be very challenging considering that they can only perform specific tasks, which also limits their applicability for now. Other challenges are to create a quantum programming language for programming Quantum logical gates (QLCs) and overcoming the quantum noise that can generate instability in the quantum computing systems and results. 
Quantum states can be easily disturbed. This disturbance on the quantum states can lead to data loss in the quantum computer environment and change the qubits results in the entire computational process. This quantum disturbance is known initially as quantum noise. The quantum noise can occur due to several sources of disturbance on the quantum states, affecting the performance of qubits. A few examples of the root cause of the quantum noise are the variance of temperature, cosmic radiation, and the influence of the external magnetic field. It is known, for example, that thermal noise can affect electric circuits at low currents [24], and this thermal noise is also found in the quantum noise properties above the interstellar temperatures [25]. This is one of the reasons why the current Quantum Computers need to be planned and operated at below or equal to interstellar temperature. This process does planning for a Quantum computer's architecture very costly due to requiring a shielded room operating close or below to the zero absolute. Another fact is that qubits disturbance occurring due to quantum noise also affects the QLCs, which prevents them from performing their task accurately [26]. The means cryo-room controls are employed to mitigate the issue related to quantum noise. Thus, Quantum computers are currently operating around interstellar temperatures and are using additional qubits for quantum noise detection and correction. It is important to notice that the closer it gets the environment's temperature to zero absolute, the molecules start acting at the zero-point of energy [27]. Thus based on the law of thermodynamics, there is no energy to be transferred between molecules based on motion, which consequently ceased the quantum noise due to thermal activities, helping to preserve the quantum states. Chart 1 below shows the comparison of Kelvin and Celsius to operate a Microsoft Quantum computer architecture.

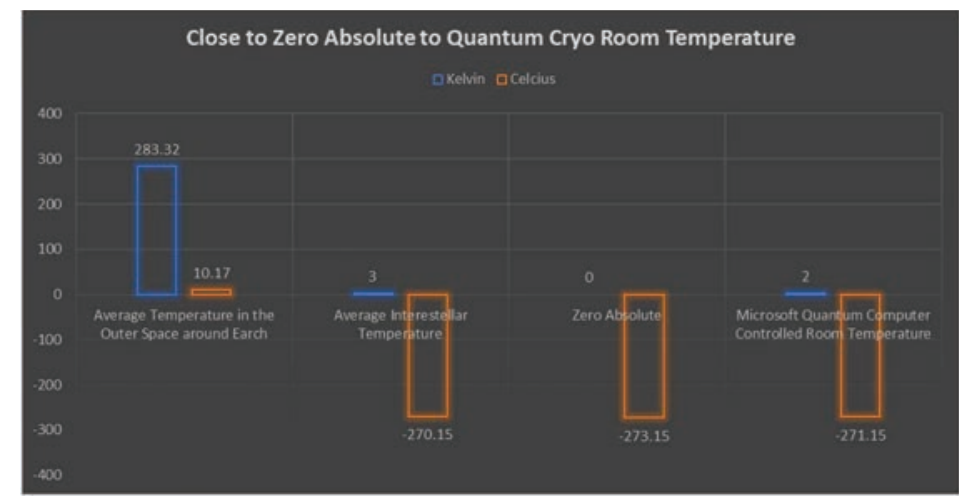

Chart 1 Microsoft quantum computer's room temperature. 
Nevertheless, some progress has been made. Presently, quantum computers exist, and the field of quantum computation is growing faster to overcome all physical barriers to control quantum physics, particularly CryoComplementary Metal Oxide Semiconductor (Cryo-CMOS) [28] circuits to emulate quantum mechanics gates. Once this is achieved, the quantum computation can have embedded in its architecture even more and more qubits power and quantum gates to achieve quantum supremacy [29].

\subsubsection{Quantum computer current state of art}

Starting from the principle of the current state of the art for Quantum computers, it is interesting to present the existing architecture created by Microsoft based on its cryo-computer core for commercially scaling up the quantum computers. As demonstrated in the figure below, Microsoft's concept created a quantum computer stack amalgamated with a classical computer stack to complement each other. For this type of quantum computer architecture, we, the authors of this paper, will propose the name of Quantum-to-Classical Computer Integrated Architecture or simply Q2CIA as presented below in Figure 6.

As can be seen from the Microsoft Quantum Computer stack designed above, from the bottom to the top, there are three main stages of integration

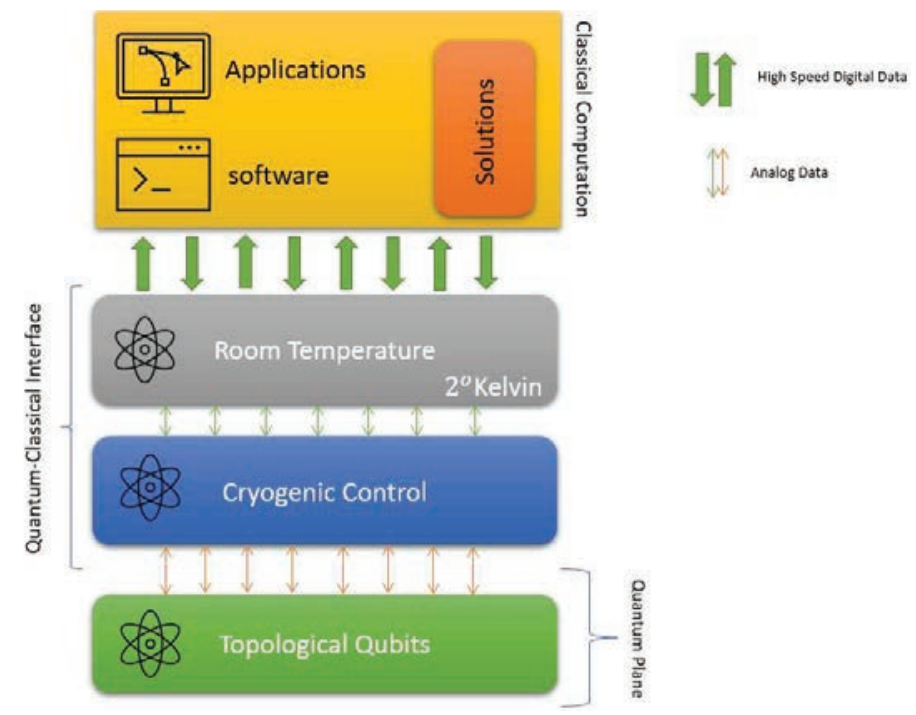

Figure 6 Microsoft topological quantum computing. 
based on the quantum computation stack to control the qubits and QLGs. The principal is based on topological qubits fabrication. This technique of using topological qubits is under investigation by Microsoft. Topology is "is the mathematical study of the properties that are preserved through deformations, twistings, and stretchings of objects." [30]. Microsoft aims to build a manufacturing process to create the next generation of advanced Qubit processors with this mathematical approach. This process is designed to create stable quantum computer processors that can be resistant to quantum noise. The first part is the Quantum Plane, where the topological Qubits states are controlled. There is an orchestration of superconductors and semiconductors to operate in this plane and quantum gates to operate "raw qubits," all at the quantum state level. The cryogenic control offers what it stands for to control the qubits preserving their states. It also represents the heart of qubits communication processes before transitioning the quantum results to the upper layer for running them in classical computation based on software and application solutions. It is important to realize that Microsoft and the University of Sydney have created a new Cryo-CMOS [31] circuit designed to operate is better at low room temperature, which dissipates less energy in a combination of digital and analog circuits. This new Cryo-CMOS circuit is being tested to facilitate the operation of multiple Qubits [32], paving the way for a more advanced Quantum computer generation that will enable the orchestration of thousands of Qubits instead of very few controlled today.

Another interesting field of quantum computing research is, for instance, circuit quantum electrodynamics (cQED). As presented earlier in the introduction, Feynman and his colleagues developed the QED theory followed by the works continued by Einstein in the scientific article famously known as the EPR paper, which enabled the concept of quantum computers. cQED is an area of quantum computer technology focused on merging quantum hardware and resonators [33] to enable process qubits. The resonators act as a standard bus connecting all qubits and making them readable. As presented in the principle of quantum mechanics, to read a value in a particle, there is a need to have quantum interference. Then the particle will collapse, and the value will be revealed. cQED seems to be a promissory area of research to make it possible to store and process qubits in a circuits board, as currently exists in a motherboard for processing classical bits in classical computers similarly to personal computers (PCs) or supercomputers. As demonstrated by Microsoft, "Building a truly scalable quantum computer involves creating raw materials for topological quantum devices, fabricating cold electronics and refrigeration systems, and developing overall infrastructure." [34] Once 
all the obstacles of creating a quantum computer are removed or overcome, it is evident that the first ones to benefit economically will be the hyperscalers. Hyperscalers will offer Quantum computing services based on Cloud services. The reason for this assumption is because they will be the ones capable of enduring a total cost of ownership (TCO) to continue investing in R\&D and scaling up for critical applications. They are currently offering quantum servers to test porpuses based on APIs to allow collaborative innovation with the global audience to shape their quantum technologies. These critical applications will not be limited to academia and the private market for communications providers. Quantum Computers in Cloud Services will encompass clients as 6G Mobile Network Operators (6G-MNOs) and the next generation of the Internet, which includes the Next Generation of Internet Service Providers (NextGen ISPs) that will finally deploy the new version of Internet Protocol version 6 (IPv6).

\subsubsection{Quantum communications and security}

Quantum Communications is another level of employing quantum mechanics to engineer solutions for high-security communication channels. With the evolution of quantum computing, security threats will also grow. Therefore, many institutions are studying the future threats posed by quantum computers in case rogue states or cybercriminals use this technology. The North American institution National Security Agency (NSA) has laid a new area of investigation denominated Post-Quantum Cyber Security Resources to focus on solutions to counter-attack or prevent the quantum menace [37]. The idea of creating a quantum network that applies quantum mechanics to utilize quantum key distribution (QKD) and generates a quantum key cryptography system for providing a safer network is paramount, and it will be responsible for creating the next generation of the Internet. This new security approach is also known as quantum-safe zero trust architecture [38]. The QKD process is different from the current public keys, which rely on the mathematics to be generated. In the QKD process, generating a key and sharing it with the trusted device is based on quantum mechanics, which creates a safer exchange of keys and is robust against security breaches.

The realization of quantum communications is made to offer a robust security network that abides by a true random exchange of digital security keys and that follows the quantum physics phenomenon. Having this type of communication technology, a perturbation on the quantum superposition will affect the qubits, and it can suspend the entire communication channel, making it almost $100 \%$ tempered proof. Nowadays, the exchange of encryption 
keys is made using classical bits, in which data is encrypted using electrical pulses of 0 or 1 or optical pulses, always obeying the physical medium characteristics. The security issue related to this process is that any hacker can read these bits "on the fly" and reveal the encrypted data or temper it in different formats of cyberattacks - also, the digital key can be broken if not robust enough. Here comes the importance of rethinking how to create a robust and safer encryption process utilizing quantum communications.

Quantum communications, in principle, follow the Quantum Shannon theory. Quantum Shannon Theory [35] is based on the studies of transmitting information in quantum systems. In summary, quantum communications sent data in classical bits but allowed to encrypt the public key utilizing quantum computation to run the QKD. The primary encryption process for QKD is the known BB84 protocol. Below is a description of Quantum Key Distribution (QKD) and an explanation for a communication channel between Alice and Bob [36]. For Alice to send protected information to Bob via a quantum communication process, here are the steps of QKD in a quantum communication system.

(1) Alice encrypts her public key number utilizing qubits in a specific polarization of photons.

(2) The Qubits are dispatched to Bob via an optical channel.

(3) If the eavesdropper tries to read Alice keys on the optical channel, it will generate a quantum noise that will collapse the Qubits via a process known as decoherence, and the only thing left is noise.

(4) Therefore, the key will be destroyed, and Bob and Alice would run a process to identify if an intrusion caused the noise. If confirmed, the original key generated by Alice is discarded, and a new one is created. This process is identified as "key distillery".

(5) Once the Alice keys are exchanged with Bob without any external intrusion, Bob calculates her key, and he gets ready to receive the encrypted data.

(6) Finally, Alice will encrypt her message using her quantum key, transmit it in a classical bit, and submit it to Bob in a safer quantum channel.

After the general explanation regarding the quantum key distribution, it follows a presentation of the Quantum communication network [37] from the quantum equipment perspective.

As presented in Figure 7 above, the Quantum network comprises quantum repeaters to allow the transmission of qubits in the communication channel, avoiding employing photons to be transported end-to-end. In this process is 


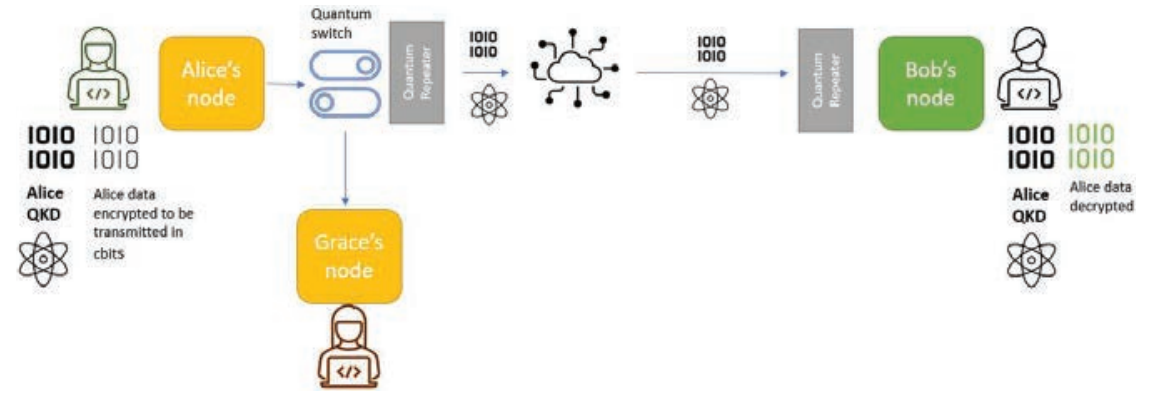

Figure 7 Quantum networks concept.

applied the quantum teleportation. Quantum repeaters use quantum teleportation to enable QKD to preserve the quantum states in the medium without using the constant optical medium to establish a quantum communication channel, which would be very costly.

As can be seen, despite all challenges, quantum communications is a promising area of research to create the next generation of safer digital communication. Several institutions and organizations are researching quantum communications services, including the pioneering project initiated by the European Space Agency (ESA) on the project baptized as Security And cryptoGrAphic mission (SAGA), in which the objectives are to create a Quantum Communication Networks (QCN) [38].

\subsubsection{Quantum machine learning for $6 \mathrm{~g}$ networks}

Fundamentally, quantum computers carry out the mathematical tasks of reversible operations [39]. Quantum Computing will support the development of innovative services, and one of the newest areas of investigation is the synergy of the quantum computer with artificial intelligence (AI). The domain of AI is a reality from now on, and then many applications are deploying AI to overcome obstacles to deliver agility and solutions for several vertical industries. One of the vertical industries profiting from AI technologies is wireless communications services, as AI is being embedded in Smartphones to cellular architecture to control network services and resources. $6 \mathrm{G}$ will deal with billions of devices, handling several types of applications and different categories of virtual networks and several Service Level Agreement (SLA) levels for all flavors of application services. Thus, $6 \mathrm{G}$ will inaugurate the era of the Internet of Beings (IoB), offering a new generation of wireless communication networks and enabling the dawn of Society 5.0, which is a new framework created by the Japanese government [40]. 
Society 5.0 technology serves humanity, creating a synergy between the cyber and physical world to deliver world-class services to support humanity at all social levels and for all economic classes to speed up ITU Connect Agenda 2030 based on the Sustainable Development Goals (SDGs) [41]. So this newly created framework will depend on an intelligent network, omnipresent, that can support Cloud Services and AI technologies to attend several services.

Thus, 6G is a crucial enabler of Society 5.0 as a backbone to achieve these goals. The biggest challenge envisaged in this future scenario will be to process the exponential growth of Big Data and to guarantee the SLA for future 6G Networks and Society 5.0 use cases. Additionally, it is essential to highlight the number of devices fully connected to the internet is expected to be 59 times larger than the world population by 2030 [42]. New technology is being studied to tackle these demands, which represents the merge of quantum computing and Machine Learning (ML). ML is a branch of artificial intelligence that tries to emulate the human brain and its learning process. Therefore, ML and Quantum Computing create a new scientific framework known as Quantum Machine Learning (QML) [43].

QML has to employ the best of QC and ML to create a new service architecture to process real-time or near real-time the learning, classification, and prioritization of data and services at exponential computing power speed never seen before. With this, QML will support the next generation of Multiple-Access Edge Computing (MEC) to create an agile and intelligent cellular edge controller. Additionally, QML will be deployed as Cloud Service $(\mathrm{CaaS})$ at the edge or in the core of the $6 \mathrm{G}$ Networks infrastructure. Recognizing the role of QML in the future 6G architecture opens up several opportunities to be used and resolve issues in the network domain with celerity and precision.

\section{Conclusions}

In conclusion, quantum mechanics applied in computing technologies will create a new generation of services that will reshape the world, creating a new industrial revolution. Maybe this new field of quantum technology will signify the beginning of Industry 5.0. Notably, there are still existing many challenges to be overcome in quantum technologies. The know-how and material to build such quantum computer architecture or quantum communications networks are not mature yet. However, progress has been made, and 
optimistically these new waves of technologies based on quantum mechanics shall be ready to be used commercially in the next five to ten years from now. Thus the continuous investment in $R \& D$ for quantum mechanics applied for computational technologies, together with Artificial Intelligence, is vital for the future of $6 \mathrm{G}$ networks. $6 \mathrm{G}$ networks infrastructure will support a brand-new era of innovation by 2030, then the synergy of both state of art technologies will create a digital bridge for a true convergence of the physical and the cyberworld. In this new era, humanity will embrace further hightech advancements created by quantum technologies and underpinned by the future wireless communication services to foster a better world, positively impacting our society and our planet. As expected, these advancements will deliver progress in all areas, from medicine to space explorations. With this approach, Society 5.0 and the SDGs will be met in the following decades.

Moreover, in the next era, the deployment of Quantum Services, especially Quantum as a Service (QaaS) via Cloud service by hyperscale's, will be pivotal for fostering the deployment of two other essential research areas beyond 2030 as CONASENSE and Knowledge Home. CONASENSE stands for integrating communications, navigation, sense, and services, and the objectives are to improve services in all fields, offering better synergy amongst these integrated parts. Knowledge Home stands for Knowledge Human Bond Communication Beyond 2050 [44]. Knowledge Home aims to integrate all humans' five senses with technology to allow them to interact with the environment bolstered by wireless technologies, computers, and Artificial Intelligence.

Finally, the fundamental question is to be answered, and it is based on the capacity of the scientific community to be able to engineer quantum computers capable of obeying the fundamental laws of computer manufacturing power and computer economics price availability as presented by the first and second Moore's Law [45] and The Law of Accelerating Returns [46], which will finally demonstrate the exponential technological Breakthru experienced by humanity in the decades to come.

\section{References}

[1] P. S. Rufino Henrique and R. Prasad, " $6 \mathrm{G}$ - The Road to the Future Wireless Technologies 2030," River Publishers: Professional Books, 31Mar-2021. [Online]. Available: https://www.riverpublishers.com/book _details.php?book_id=920. [Accessed: 27-Jul-2021]. 
[2] "Quantum mechanics," Encyclopedia Britannica. [Online]. Available: ht tps://www.britannica.com/science/quantum-mechanics-physics. [Accessed: 28-Jul-2021].

[3] C. Orzel, "Six things everyone should know about quantum physics," Forbes, 08-Jul-2015. [Online]. Available: https://www.forbes.com/sites /chadorzel/2015/07/08/six-things-everyone-should-know-about-quantu m-physics/?sh=c77facb7d467. [Accessed: 28-Jul-2021].

[4] New Scientist, "Quantum physics," New Scientist. [Online]. Available: https://www.newscientist.com/definition/quantum-physics/. [Accessed: 28-Jul-2021].

[5] C. Gearhart, "Compendium of quantum physics |SpringerLink," Springer, 2009. [Online]. Available: https://link.springer.com/book/1 0.1007/978-3-540-70626-7. [Accessed: 28-Jul-2021].

[6] "The Nobel Prize in Physics 1918 - Max Planck Nobel Lecture," NobelPrize.org. [Online]. Available: https://www.nobelprize.org/prizes/phys ics/1918/planck/lecture/. [Accessed: 28-Jul-2021].

[7] V. Mott, "Introduction to chemistry," Lumen. [Online]. Available: https: //courses.lumenlearning.com/introchem/chapter/plancks-quantum-theo ry/. [Accessed: 28-Jul-2021].

[8] "The Nobel Prize in Physics 1922 - Niels Bohr Facts," NobelPrize.org. [Online]. Available: https://www.nobelprize.org/prizes/physics/1922/b ohr/biographical/. [Accessed: 28-Jul-2021].

[9] "The Nobel Prize in physics 1932 - Werner Heisenberg Biographical," NobelPrize.org. [Online]. Available: https://www.nobelprize.org/prizes/ physics/1932/heisenberg/biographical/. [Accessed: 28-Jul-2021].

[10] J. Hilgevoord and J. Uffink, "The uncertainty principle," Stanford Encyclopedia of Philosophy, 12-Jul-2016. [Online]. Available: https://plato. stanford.edu/entries/qt-uncertainty/. [Accessed: 28-Jul-2021].

[11] A. Einstein , B. Yakovlevich Podolsky , and N. Rosen , "Can QuantumMechanical Description of Physical Reality be Considered Complete?," CERN, 15-May-1935. [Online]. Available: http://cds.cern.ch/record/4 05662/files/PhysRev.47.777.pdf. [Accessed: 28-Jul-2021].

[12] A. Chodos, J. Ouellette, and E. Tretkoff, "This month in physics history," American Physical Society, Nov-2005. [Online]. Available: https://ww w.aps.org/publications/apsnews/200511/history.cfm. [Accessed: 28-Jul2021].

[13] J. S. Bell, "On the einstein podolsky rosen paradox* - CERN," CERN, 04-Nov-1964. [Online]. Available: https://cds.cern.ch/record/111654/fil es/vol1p195-200_001.pdf. [Accessed: 06-Nov-2021]. 
[14] B. Brubaker, "How bell's theorem proved 'Spooky action at a distance' is ...," Quantamagazine, 20-Jul-2021. [Online]. Available: https://ww w.quantamagazine.org/how-bells-theorem-proved-spooky-action-at-a -distance-is-real-20210720/. [Accessed: 06-Nov-2021].

[15] A. Whitaker, "John Bell and the most profound discovery of science," Physics World, 01-Dec-1988. [Online]. Available: https://physicsworld .com/a/john-bell-profound-discovery-science/. [Accessed: 06-Nov2021].

[16] "The Nobel Prize in physics 1965," NobelPrize.org. [Online]. Available: https://www.nobelprize.org/prizes/physics/1965/summary/. [Accessed: 28-Jul-2021].

[17] Benioff, Paul. "The computer as a physical system: A microscopic quantum mechanical Hamiltonian model of computers as represented by Turing machines," 1980. [Online]. Available: https://www.research gate.net/profile/Paul-Benioff/publication/226754042_The_computer_a s_a_physical_system_A_microscopic_quantum_mechanical_Hamiltonian _model_of_computers_as_represented_by_Turing_machines/links $/ 55 \mathrm{c} 2$ 360708aeb975673e3dce/The-computer-as-a-physical-system-A-micro scopic-quantum-mechanical-Hamiltonian-model-of-computers-as-repr esented-by-Turing-machines.pdf. [Accessed: 28-Jul-2021].

[18] H.-A. Bachor and M. Simmons, "Quantum computing," Australian Academy of Science, 23-Nov-2017. [Online]. Available: https://ww w.science.org.au/curious/technology-future/quantum-computing. [Accessed: 28-Jul-2021].

[19] Quantiki, "Shor's factoring algorithm," Quantiki. [Online]. Available: https://www.quantiki.org/wiki/shors-factoring-algorithm. [Accessed: 06-Nov-2021].

[20] European Commission, "Quantum technologies flagship," Shaping Europe's digital future. [Online]. Available: https://digital-strategy.e c.europa.eu/en/policies/quantum-technologies-flagship. [Accessed: 28-Jul-2021].

[21] E. Gibney, "Hello quantum world! Google publishes landmark quantum Supremacy claim," Nature News, 23-Oct-2019. [Online]. Available: ht tps://www.nature.com/articles/d41586-019-03213-z. [Accessed: 28Jul-2021].

[22] "Quantum computing," Microsoft Research, 01-Feb-2021. [Online]. Available: https://www.microsoft.com/en-us/research/research-area/q uantum-computing/?facet $\% 5 \mathrm{Btax} \% 5 \mathrm{D} \% 5 \mathrm{Bmsr}$-research-area $\% 5 \mathrm{D} \% 5$ B0\%5D=243138\&amp;sort_by=most-recent. [Accessed: 28-Jul-2021]. 
[23] C. Fisher, "IBM: Quantum computing," IBM Quantum, 02-Apr-2009. [Online]. Available: https://www.ibm.com/quantum-computing/. [Accessed: 28-Jul-2021].

[24] J. Andersson and M. Johansson, "Emulated quantum noise," Emulated quantum noise - Emulated quantum noise - CSC Company Site, 09Mar-2021. [Online]. Available: https://www.csc.fi/en/-/emulated-quan tum-noise. [Accessed: 06-Nov-2021].

[25] K. Haynes, "How cold is it in outer space?," Discover Magazine, 04May-2020. [Online]. Available: https://www.discovermagazine.com/the -sciences/how-cold-is-it-in-outer-space. [Accessed: 07-Nov-2021].

[26] A. Libal, "The temperatures of outer space around the Earth," Sciencing, 02-Mar-2019. [Online]. Available: https://sciencing.com/temperatures -outer-space-around-earth-20254.html. [Accessed: 07-Nov-2021].

[27] Encyclopedia Britannica, "Absolute zero," Encyclopedia Britannica. [Online]. Available: https://www.britannica.com/science/absolute-zero. [Accessed: 07-Nov-2021].

[28] S. J. Pauka, K. Das, R. Kalra, A. Moini, Y. Yang, M. Trainer, A. Bousquet, C. Cantaloube, N. Dick, G. C. Gardner, M. J. Manfra, and D. J. Reilly, "A cryogenic CMOS chip for generating control signals for multiple qubits," Nature News, 25-Jan-2021. [Online]. Available: https://www.nature.com/articles/s41928-020-00528-y. [Accessed: 07-Nov-2021].

[29] P. S. Rufino Henrique, "Quantum Physics applied for the future architecture of the 6G Networks," The 6G Hybrid Workshop - CGC and Aarhus University, 12-Oct-2021. [Online]. Available: https://ctifglobalcapsule. org/wp/wp-content/uploads/docs/events/6GNetworks-PauloHenrique.p df. [Accessed: 06-Nov-2021].

[30] "Topology," Wolfram MathWorld. [Online]. Available: https://mathworl d.wolfram.com/Topology.html. [Accessed: 07-Nov-2021].

[31] C. Nayak, "Full stack ahead: Pioneering quantum hardware allows for controlling up to thousands of qubits at cryogenic temperatures," Microsoft Research, 27-Jan-2021. [Online]. Available: https://www.mi crosoft.com/en-us/research/blog/full-stack-ahead-pioneering-quantumhardware-allows-for-controlling-up-to-thousands-of-qubits-at-cryogen ic-temperatures/. [Accessed: 07-Nov-2021].

[32] S. J. Pauka, K. Das, R. Kalra, A. Moini, Y. Yang, M. Trainer, A. Bousquet, C. Cantaloube, N. Dick, G. Gardner, M. J. Manfra, and D. Reilly, "A cryogenic CMOS chip for generating control signals for multiple qubits," Microsoft Research, 26-Jan-2021. [Online]. Available: 
https://www.microsoft.com/en-us/research/publication/a-cryogen ic-cmos-chip-for-generating-control-signals-for-multiple-qubits/. [Accessed: 07-Nov-2021].

[33] T. Q. Team, "Circuit quantum electrodynamics," Qiskit, 26-Jul-2021. [Online]. Available: https://qiskit.org/textbook/ch-quantum-hardware/ cQED-JC-SW.html. [Accessed: 28-Jul-2021].

[34] "Quantum technology: Microsoft Azure," Quantum Technology |Microsoft Azure. [Online]. Available: https://azure.microsoft.com/en-u s/solutions/quantum-computing/technology/. [Accessed: 28-Jul-2021].

[35] M. M. Wilde, "Concepts in quantum shannon theory (Chapter 1) quantum information theory," Cambridge Core. [Online]. Available: https://www.cambridge.org/core/books/abs/quantum-information-t heory/concepts-in-quantum-shannon-theory/DB95B7F9D7FEDF6FA 4E0150A60C56B32. [Accessed: 07-Nov-2021].

[36] M. Giles, "Explainer: What is Quantum Communication?," MIT Technology Review, 02-Apr-2020. [Online]. Available: https://www.technolo gyreview.com/2019/02/14/103409/what-is-quantum-communications/. [Accessed: 07-Nov-2021].

[37] OFCOM, "Quantum Communications: New potential for ... - ofcom. org.uk," Ofcom, 28-Jul-2021. [Online]. Available: https://www.ofcom. org.uk/_data/assets/pdf_file/0013/222601/Executive-Summary.pdf. [Accessed: 07-Nov-2021].

[38] P. S. Rufino Henrique and R. Prasad, "The Road for 6G Multimedia Applications," 2020 23rd International Symposium on Wireless Personal Multimedia Communications (WPMC), 2020, pp. 1-6, doi: 10.1109/WPMC50192.2020.9309478.

[39] "Quantum computing for computer scientists," Microsoft Research, 14May-2018. [Online]. Available: https://www.microsoft.com/en-us/rese arch/video/quantum-computing-computer-scientists/\#!related_info. [Accessed: 07-Nov-2021].

[40] Cabinet Office, Society 5.0. [Online]. Available: https://www8.cao.go. jp/cstp/english/society5_0/index.html. [Accessed: 28-Jul-2021].

[41] UN-ITU. "Connect 2030 - an agenda to connect all to a better world," ITU. [Online]. Available: https://www.itu.int/en/mediacentre/backgro unders/Pages/connect-2030-agenda.aspx. [Accessed: 12-Jul-2021].

[42] M. Kuipers, "6G The Last Generation ," CGC, 12-Oct-2021. [Online]. Available: https://ctifglobalcapsule.org/wp/wp-content/uploads/docs/e vents/6gworkshop-Martijn-Kuipers.pdf. [Accessed: 07-Nov-2021]. 
[43] H.-Y. Huang, M. Broughton, M. Mohseni, R. Babbush, S. Boixo, H. Neven, and J. R. McClean, "Power of data in quantum machine learning," Nature News, 11-May-2021. [Online]. Available: https://www.na ture.com/articles/s41467-021-22539-9. [Accessed: 28-Jul-2021].

[44] R. Prasad, "Knowledge home," 2016 International Conference on Advanced Computer Science and Information Systems (ICACSIS), 2016, pp. 33-38, doi: 10.1109/ICACSIS.2016.7872717.

[45] Intel, "Over 50 years of Moore's law," Intel. [Online]. Available: https: //www.intel.com/content/www/us/en/silicon-innovations/moores-law-t echnology.html. [Accessed: 06-Nov-2021].

[46] R. Kurzweil, "The Law of Accelerating Returns," Kurzweil The Law of Accelerating Returns Comments. [Online]. Available: https://www.kurz weilai.net/the-law-of-accelerating-returns. [Accessed: 06-Nov-2021].

\section{Biographies}

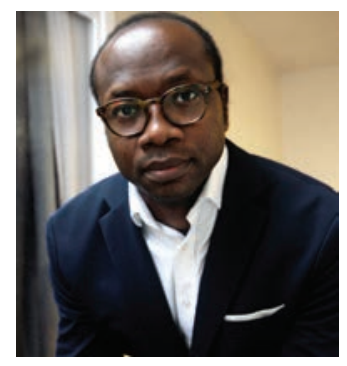

Paulo Sergio Rufino Henrique (Spideo -Paris, France). CTIF Global Capsule, Department of Business Development and Technology, also Aarhus University, Herning, Denmark. Paulo S. Rufino Henrique holds more than 20 years of experience working in telecommunications. His career began as a field engineer at UNISYS in Brazil, where he was born. There, Paulo worked for almost nine years in the Service Operations, repairing and installing corporative servers and networks before joining British Telecom (BT) Brazil. Paulo worked five years at BT Brazil managing MPLS networks, satellites (V-SAT), IP-Telephony for Tier 1 network operations. During that period, he became the Global Service Operations Manager overseeing BT operations in EMEA, Americas, India, South Korea, South African, and China. After a successful career in Brazil, Paulo got transferred to the BT headquarters in London, where he worked for six and a half years as a service manager for Consumers Broadband in the UK and IPTV Ops manager for BT TV 
Sports channel. Additionally, during his tenure as IPTV Ops manager for BT, Paulo also participated in the BT project of launching the first UHD (4K) TV channel in the UK. He then joined Vodafone UK as a Quality Manager for Consumers Broadband Services and OTT platforms, and he worked in that capacity for almost two years. During his stay in London, Paulo completed a Post-graduation Degree at Brunel London University. His thesis was entitled 'TV Everywhere and the Streaming of UHD TV over 5G Networks \& Performance Analysis'. Presently, Paulo Henrique holds the Head of Delivery and Operations position at Spideo, Paris, France. He is also a Ph.D. candidate under Professor Ramjee Prasad's supervision at Global CTIF Capsule, Department of Business at Aarhus University, Denmark. His research field is 6G Networks - Performance Analysis for Mobile Multimedia Services for the Future Wireless Technologies.

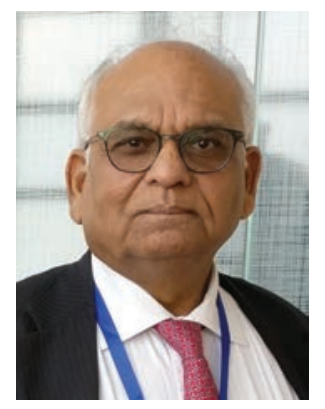

Ramjee Prasad, CTIF Global Capsule, Department of Business Development and Technology, also Aarhus University, Herning Denmark. Dr. Ramjee Prasad, Fellow IEEEIEEE, IET, IETE, and WWRF, is a Professor of Future Technologies for Business Ecosystem Innovation (FT4BI) in the Department of Business Development and Technology, Aarhus University, Herning, Denmark. He is the Founder President of the CTIF Global Capsule (CGC). He is also the Founder Chairman of the Global ICTICT Standardization Forum for India, established in 2009. He has been honored by the University of Rome "Tor Vergata", Italy as a Distinguished Professor of the Department of Clinical Sciences and Translational Medicine on March 15, 2016. He is an Honorary Professor of the University of Cape Town, South Africa, and the University of KwaZulu-Natal, South Africa. He has received the Ridderkorset of Dannebrogordenen (Knight of the Dannebrog) in 2010 from the Danish Queen for the internationalization of top-class telecommunication research 
and education. He has received several international awards such as IEEE Communications Society Wireless Communications Technical Committee Recognition Award in 2003 for making a contribution in the field of "Personal, Wireless and Mobile Systems and Networks", Telenor's Research Award in 2005 for impressive merits, both academic and organizational within the field of wireless and personal communication, 2014 IEEE AESS Outstanding Organizational Leadership Award for: "Organizational Leadership in developing and globalizing the CTIF (Center for TeleInFrastruktur) Research Network", and so on. He has been the Project Coordinator of several EC projects, namely, MAGNET, MAGNET Beyond, eWALL. He has published more than 50 books, 1000 plus journal and conference publications, more than 15 patents, over 140 Ph.D. Graduates and a larger number of Masters (over 250). Several of his students are today worldwide telecommunication leaders themselves. 